\title{
Deposition and Characterization of Multilayer DLC/BN Films
}

\author{
Honei Chin 1 , Chavin Jongwannasiri ${ }^{2 *}$, Shuichi Watanabe ${ }^{3}$ \\ ${ }^{1}$ Graduate School of Mechanical Systems Engineering, Nippon Institute of Technology, Saitama, Japan \\ ${ }^{2}$ Ratchaburi Learning Park, King Mongkut's University of Technology Thonburi, Ratchaburi, Thailand \\ ${ }^{3}$ Department of Innovative Systems Engineering, Nippon Institute of Technology, Saitama, Japan \\ Email: *chavin.jon@kmutt.ac.th
}

How to cite this paper: Chin, H., Jongwannasiri, C. and Watanabe, S. (2017) Deposition and Characterization of Multilayer DLC/BN Films. Materials Sciences and Applications, 8, 738-745.

https://doi.org/10.4236/msa.2017.810053

Received: August 1, 2017

Accepted: September 11, 2017

Published: September 14, 2017

Copyright $\odot 2017$ by authors and Scientific Research Publishing Inc. This work is licensed under the Creative Commons Attribution International License (CC BY 4.0).

http://creativecommons.org/licenses/by/4.0/ (c) (i) Open Access

\begin{abstract}
In this article, the results obtained from a study on multilayer diamond-like carbon and boron nitride (DLC/BN) films are reported. The microstructure, atomic concentration, hardness and friction coefficient of the films were characterized using transmission electron microscopy, auger electron microscopy, nano-indentation measurements and ball-on-disk friction testing. The effects of bilayer thickness and substrate bias on film growth were investigated. All multilayer films showed alternate DLC and BN layers, except the 2- and 4-nm bilayer of multilayer DLC/BN films deposited without substrate bias. Although the layers were very thin, each layer was distinguishable. This was confirmed by the use of TEM imaging and AES measurements. The hardness values of all the multilayer films were lower than those measured for the monolayer DLC and BN films. However, the hardness can be altered with a change in the bilayer thickness. Furthermore, in the case of the films deposited with substrate bias, multilayer DLC/BN films showed an improvement in wear resistance compared to monolayer DLC and BN films. Thus, the deposition of multilayer $\mathrm{DLC} / \mathrm{BN}$ films can be considered to be beneficial in prolonging the service life of the surface.
\end{abstract}

\section{Keywords}

DLC/BN, Multilayer, RF Sputtering

\section{Introduction}

A hard, low-friction and wear-resistant film is desirable for the reduction of friction force fluctuations and atomic-scale wear. Carbonaceous films such as diamond-like carbon (DLC) films are potential candidates, with good wear resis- 
tance and lubrication properties [1] [2]. Due to the high intrinsic compressive stress, however, DLC films are easy to fracture or even peel off the substrate, which limits their wide application [3] [4]. A multilayer structure can be used to maintain the mechanical properties of the DLC while reducing the internal stresses [5] [6]. Multilayer films have been successfully demonstrated in various applications, especially for wear protection [7] [8]. Most studies have focused on the multilayer metal/DLC and ceramic/DLC films [8] [9]. There are only a few studies on the structural and mechanical properties of multilayer DLC/BN films. The structural and mechanical properties of multilayer films depend on the composition, number of interfaces and bilayer thickness [10]. A large number of internal interfaces aligned parallel to the substrate surface can retard crack propagation and provide barriers to dislocation movement [11].

In this study, a series of multilayer DLC/BN films, with different bilayer thicknesses, were prepared on a Si (100) wafer. The effects of bilayer thickness and substrate bias during film growth on the mechanical and tribological properties of the multilayer DLC/BN films have been investigated.

\section{Experimental Details}

The multilayer DLC/BN films were deposited on $\mathrm{Si}$ (100) wafers using an unbalanced RF magnetron sputtering technique. The RF sputtering apparatus is able to supply high-frequency power of $13.56 \mathrm{MHz}$ to both target and substrate, as shown in Figure 1(a). High purity graphite (99.999\%) and hexagonal boron nitride (99\%) disks of $100 \mathrm{~mm}$ in diameter and $5 \mathrm{~mm}$ in thickness were used as targets. A film was deposited by rotating a substrate at a rotation speed of $3 \mathrm{rpm}$. Before the deposition, the substrate and targets were sputter-cleaned with $\mathrm{Ar}^{+}$to remove residual surface contaminants and surface oxides using an RF power of $100 \mathrm{~W}$. The boron nitride (BN) layer was deposited first, and then the diamond-like carbon (DLC) layer was deposited. The topmost of themultilayer DLC/BN film was deposited to be a DLC layer, as shown in Figure 1(b). In this study, 2-, 4-, 6and 10-nm bilayer of multilayer DLC/BN films were deposited by controlling the number of rotating cycles. The targets were applied with an RF power of $300 \mathrm{~W}$. The negative DC bias voltage applied to the substrate was 0 and $30 \mathrm{~V}$. The deposition pressure was also set to $0.3 \mathrm{~Pa}$.

The properties of the films were studied using several characterization techniques. The structure of the multilayer DLC/BN films was evaluated by transmission electron microscopy (Hitachi HD-2000STEM, accelerating voltage; 200 $\mathrm{kV}$ ). The atomic concentration was evaluated by Auger electron spectroscopy (JANP-7800F). The hardness was measured using a nano-indentation hardness tester (Hysitron, Inc.). A diamond indenter (Berkovich-type) with an indentation load of $1000 \mu \mathrm{N}$ was used in this study. The friction coefficient was assessed using ball-on-disk friction testing (CSEM; Tribotester). A dry sliding test was performed using a ball indenter, AISI440C (SUS440C, diameter of $6.0 \mathrm{~mm}$ ) under a normal applied load of $3 \mathrm{~N}$, a rotation radius of $3 \mathrm{~mm}$, a linear speed of 


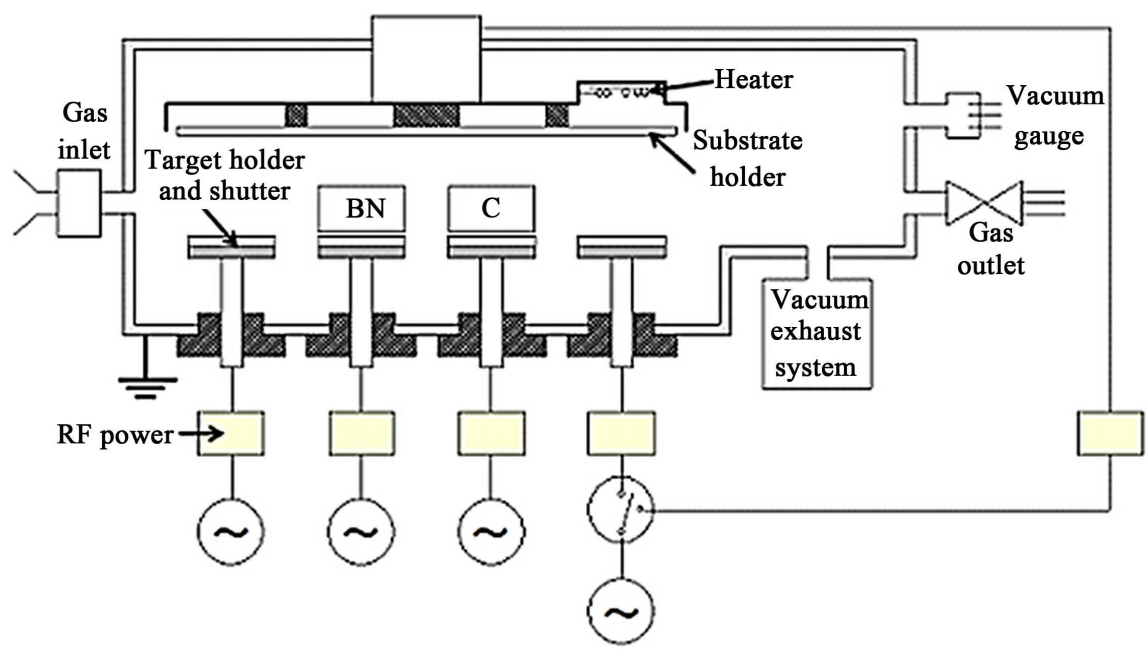

(a)

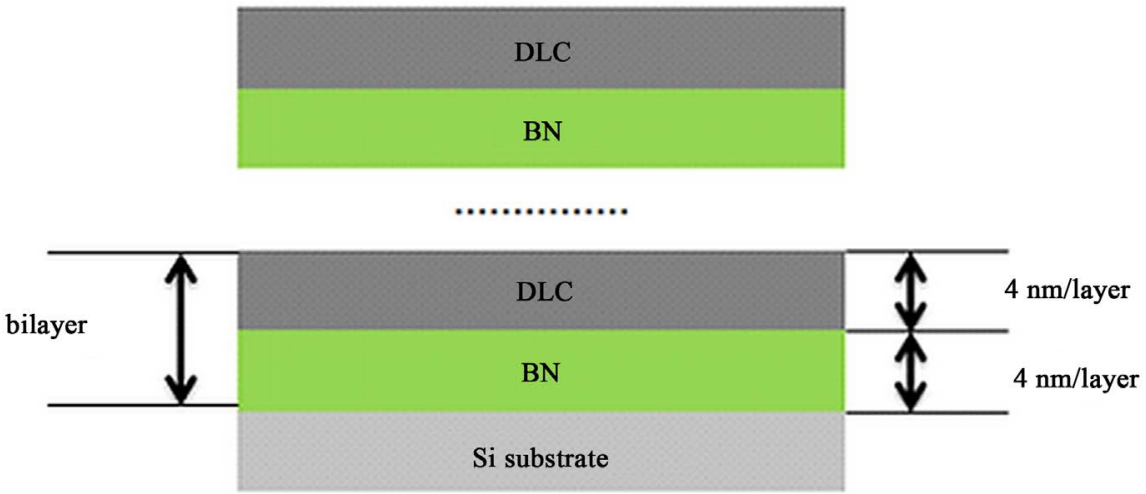

(b)

Figure 1. (a) Schematic of the unbalanced RF magnetron sputtering apparatus used in this experiment; and (b) Model of a 4-nm bilayer of a multilayer DLC/BN film.

$31.4 \mathrm{~mm} / \mathrm{s}$, and 6000 frictional rotations. The tests were performed under ambient air at room temperature.

\section{Results and Discussion}

The microstructure of the multilayer DLC/BN films was characterized by TEM analysis. Figure 2 shows TEM images obtained for a 4 -nm-thick bilayer of a multilayer DLC/BN film composed of alternate DLC and BN layers; the structure of this film was investigated in detail. The thicknesses of the DLC and BN layers shown in this TEM image are 4.5 and $3.5 \mathrm{~nm}$, respectively. This is close to the expected thickness. Although the layers are very thin, each individual layer can be distinguished. Furthermore, as shown in Figure 3, the atomic concentrations of boron, carbon and nitrogen (classified as superhard film materials) were determined by AES measurement of the depth profile of a 4-nm bilayer of a multilayer DLC/BN film. The multilayer DLC/BN films investigated in this study were deposited using the same deposition method as that indicated above. By controlling the deposition time and using different targets, the multilayer 


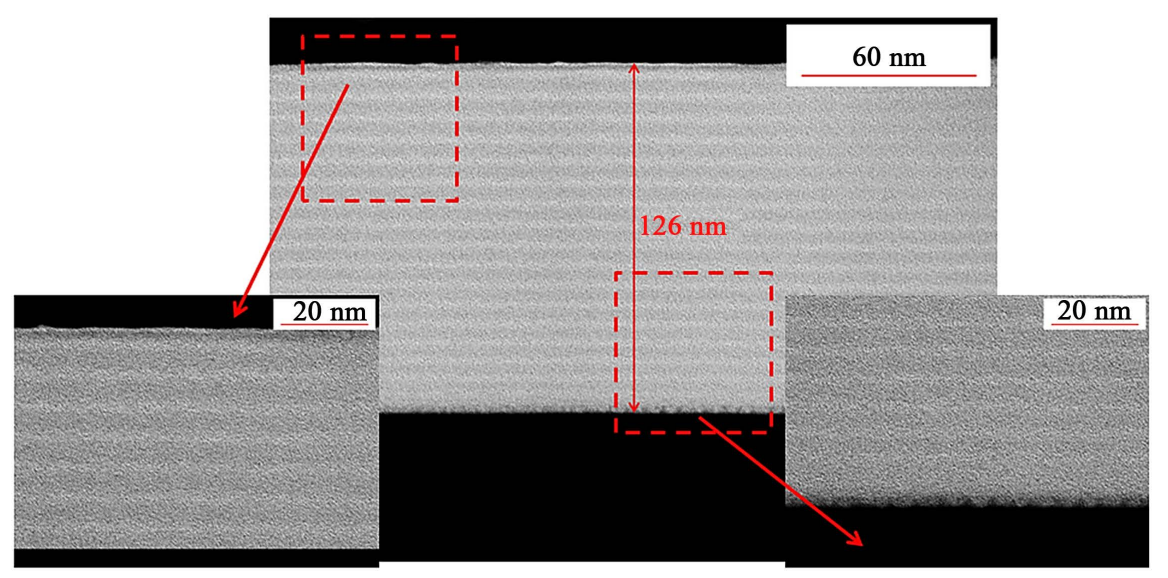

Figure 2. Cross-sectional TEM images of a 4-nm bilayer of a multilayer DLC/BN film.

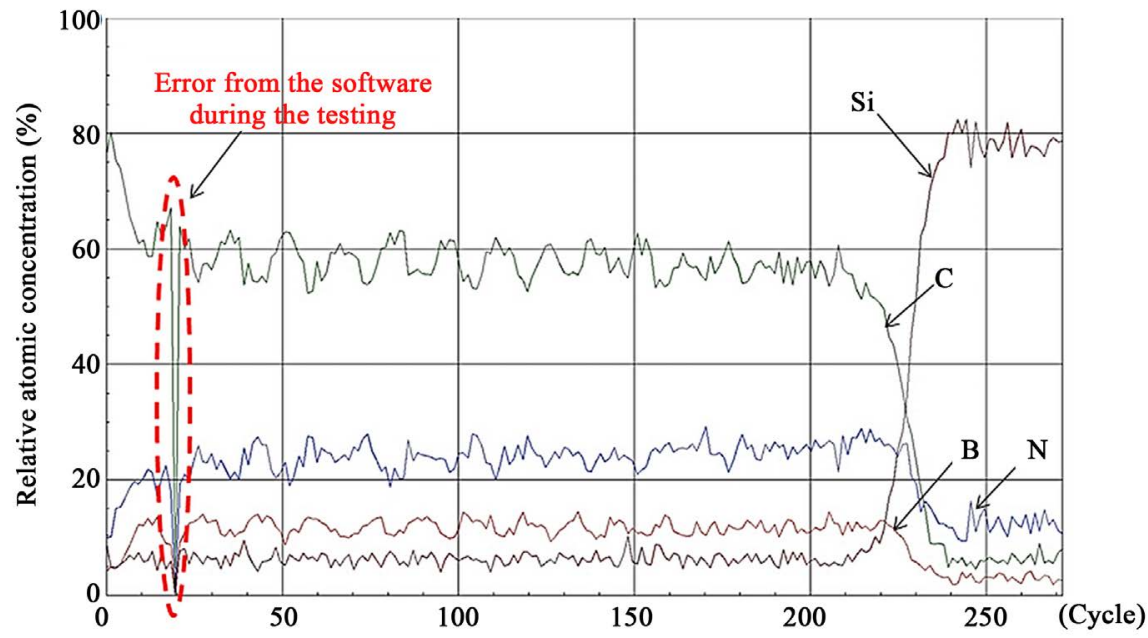

Figure 3. AES profiles of a 4-nm bilayer of a multilayer DLC/BN film.

DLC/BN films were deposited as expected. However, the 2- and 4-nm bilayers of the multilayer DLC/BN films deposited without substrate bias peeled off from the substrate one week after the deposition. This is possibly due to a high residual stress, indicating poor adhesion between the film and substrate. Therefore, it is important to consider the role of substrate bias during film growth. Li et al. found that film growth using substrate bias resulted in high-quality multilayer coatings, with low residual stress and high adhesive strength [12]. This highlights the significance of substrate bias during the deposition.

The hardness values of monolayer DLC and BN and multilayer DLC/BN films calculated from a load-displacement curve are shown in Figure 4. It is well known that a difference in the modulus between two layered materials can increase the hardness of a multilayer film [13]. Consequently, the formation of a nanoscale multilayer film composed of alternate DLC and BN layers is expected to lead to a significant increase in the hardness value compared to the monolayer film. As shown in Figure 4, however, the hardness values of all multilayer films were lower than those of the monolayer DLC and BN films. This finding contradicts 


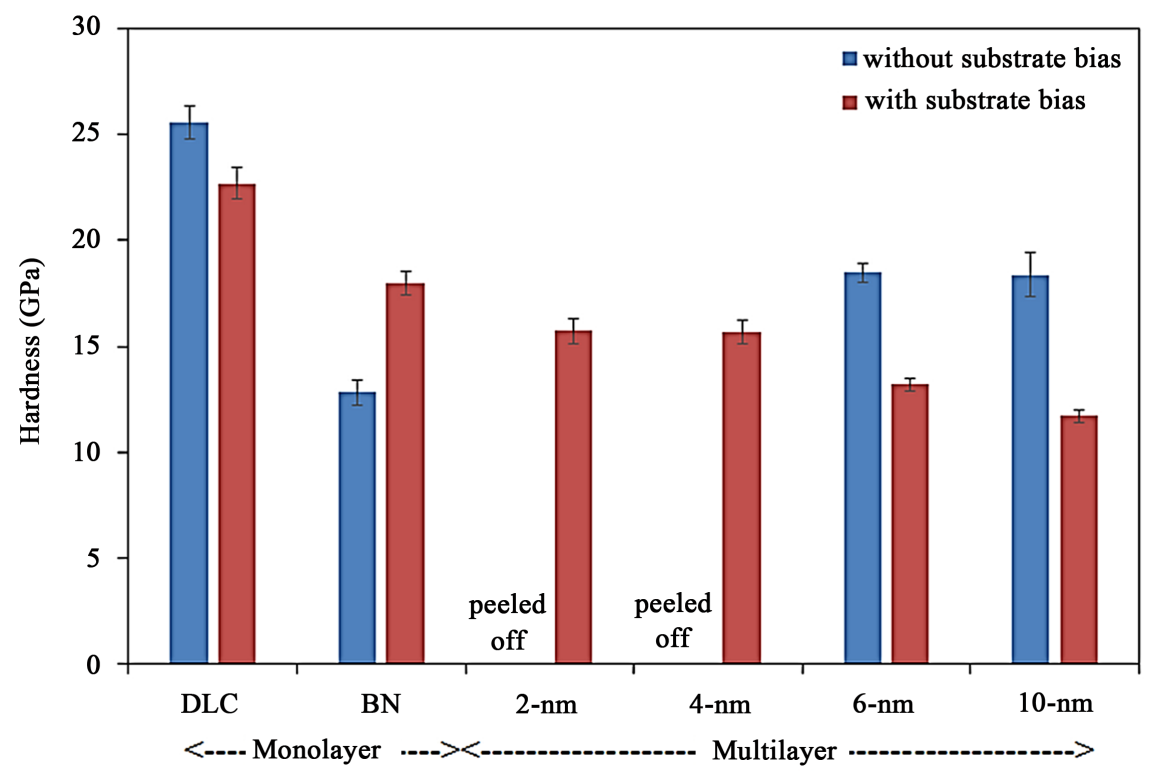

Figure 4. Hardness values of monolayer DLC and BN and multilayer DLC/BN films.

our hypothesis that the hardness value of a multilayer film is higher than that of a monolayer film.

The reason that the hardness values of the multilayer DLC/BN films were lower than those of the monolayer DLC and BN films is not clear; repeated measurements showed the same result. However, it was observed that the hardness values of the multilayer DLC/BN films deposited with a substrate bias can change with a change in bilayer thickness, whereas the multilayer films deposited without substrate bias showed no such change.

The friction coefficients of monolayer DLC and BN and multilayer DLC/BN films, all deposited with substrate bias and measured under ambient air, are shown in Figure 5. The films deposited without substrate bias were not considered in this study because some of these films peeled off from the substrate one week after the deposition. As shown in Figure 5, the wear resistance of the monolayer DLC and BN films was determined to be similar, although both films showed dissimilar values for the friction coefficients of approximately 0.18 and 0.75 , respectively. The friction coefficients of the monolayer DLC and BN films showed a simultaneous increase and fluctuations following 1000 sliding cycles. On the other hand, the multilayer DLC/BN films showed lower values for the friction coefficients than did the pure BN but higher values than the pure DLC. The friction coefficients for the 2- and 10-nm bilayer and 4- and 6-nm bilayer of the multilayer DLC/BN films were determined to be approximately 0.25 and 0.35 , respectively. However, the wear resistance of the 4- and 6-nm bilayer was higher than that of the 2- and 10-nm bilayer of the multilayer DLC/BN films. The friction coefficients of the 4- and 6-nm bilayer of the multilayer DLC/BN films were stable until approximately 5000 sliding cycles, after which they simultaneously increased and fluctuated, while the friction coefficients of the 2- and 10 -nm bilayer of the multilayer DLC/BN films were stable until approximately 


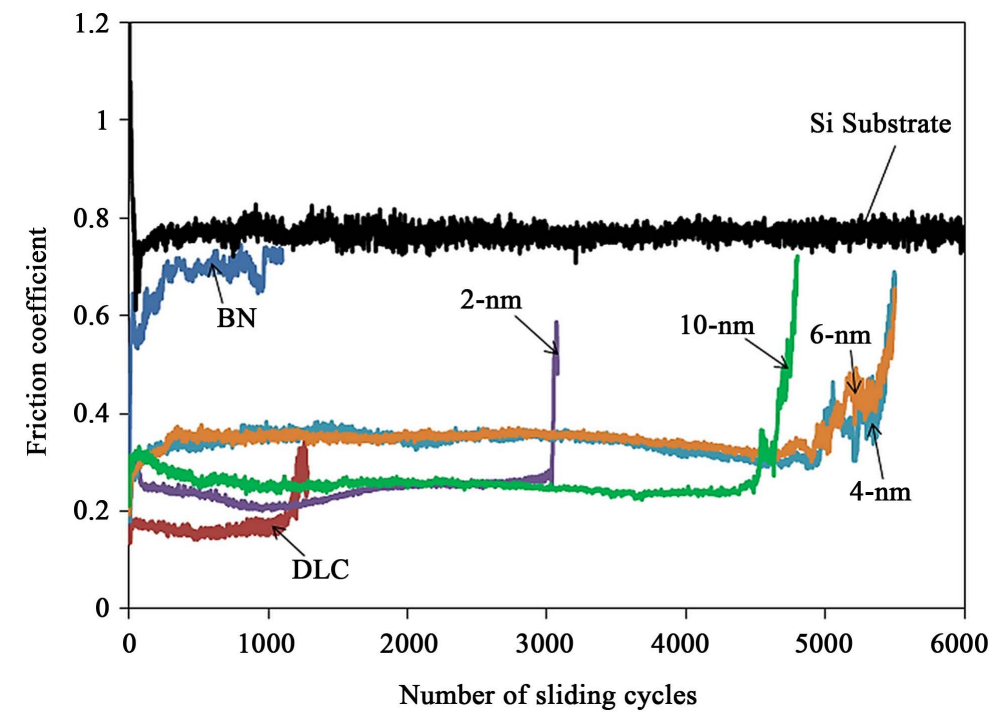

Figure 5. Friction coefficients of monolayer DLC and BN and multilayer DLC/BN films measured under ambient air.

3000 and 4500 sliding cycles, respectively. These results indicate that the wear resistance was improved due to the microstructure formed with an appropriate bilayer thickness.

The reason the multilayer DLC/BN films exhibited a longer life is considered to be as follows. For the monolayer films, the DLC or BN surface was lubricated by the monolayer itself during the friction test. Since the monolayer DLC and BN films have a poor elastic deformation, i.e., a high hardness, they can be easily removed by the friction test. In contrast, the multilayer DLC/BN films show low hardness values, and defect elongation was prevented by the interface formed between layers; therefore, deep plastic deformation is negligible, resulting in improved wear resistance [14] [15]. In addition, shearing damage due to friction was suppressed by the extremely thin layer. Based on these observations, the long service life of the surface of multilayer DLC/BN films is attributed to the multilayer microstructure.

\section{Conclusion}

Multilayer DLC/BN films were successfully synthesized on Si (100) wafers using the unbalanced RF magnetron sputtering technique. The films were investigated to determine the effects of alternating bilayer thickness and use of substrate bias during film growth on the mechanical and tribological properties. The micro-structure of the multilayer DLC/BN films reflected the composition of alternate DLC and BN layers, except for the 2- and 4-nm bilayer of multilayer DLC/BN films deposited without substrate bias. The hardness values of all the multilayer films were lower than those of the monolayer DLC and BN films. The substrate bias also affected the hardness value through a change in the bilayer thickness. Furthermore, the wear resistance of the monolayer DLC and BN films was improved by the deposition of multilayer DLC/BN films. This is due to the 
poor elastic deformation of the monolayer films and inhibition of shearing damage by the multilayer films. For the aforementioned reasons, it can be concluded that multilayer DLC/BN films can be considered to be beneficial for improving wear resistance.

\section{References}

[1] Miyake, S., Kaneko, R., Kikuya, Y. and Sugimoto, I. (1991) Micro-Tribological Studies on Fluorinated Carbon Films. Journal of Tribology, 113, 384-389.

https://doi.org/10.1115/1.2920633

[2] Miyake, S. and Kaneko, R. (1992) Microtribological Properties and Potential Applications of Hard, Lubricating Coatings. Thin Solid Films, 212, 256-261. https://doi.org/10.1016/0040-6090(92)90530-O

[3] Robertson, J. (2002) Diamond-Like Amorphous Carbon. Materials Science and Engineering: $R$, 37, 129-281. https://doi.org/10.1016/S0927-796X(02)00005-0

[4] Zou, C.W., Wang, H.J., Feng, L. and Xue, S.W. (2013) Effects of Cr Concentrations on the Microstructure, Hardness, and Temperature-Dependent Tribological Properties of Cr-DLC Coatings. Applied Surface Science, 286, 137-141. https://doi.org/10.1016/j.apsusc.2013.09.036

[5] Bull, S.J. and Jones, A.M. (1996) Multilayer Coatings for Improved Performance. Surface \& Coatings Technology, 78, 173-184. https://doi.org/10.1016/0257-8972(94)02407-3

[6] Voevodin, A.A., Schneider, J.M., Rebholz, C. and Matthews, A. (1996) Multilayer Composite Ceramicmetal-DLC Coatings for Sliding Wear Applications. Tribology International, 29, 559-570. https://doi.org/10.1016/0301-679X(95)00121-J

[7] Hu, Y., Li, L., Cai, X., Chen, Q. and Chu, P.K. (2007) Mechanical and Tribological Properties of TiC/Amorphous Hydrogenated Carbon Composite Coatings Fabricated by DC Magnetron Sputtering with and without Sample Bias. Diamond \& Related Materials, 16, 181-186. https://doi.org/10.1016/j.diamond.2006.05.010

[8] Wang, Y., Wang, J., Zhang, G., Wang, L. and Yan, P. (2012) Microstructure and Tribology of TiC(Ag)/a-C:H Nanocomposite Coatings Deposited by Unbalanced Magnetron Sputtering. Surface \& Coatings Technology, 206, 3299-3308.

https://doi.org/10.1016/j.surfcoat.2012.01.036

[9] Lin, Y.H., Lin, H.D., Liu, C.K., Huang, M.W., Chen, J.R. and Shih, H.C. (2010) Structure and Characterization of the Multilayered Ti-DLC Films by FCVA. Diamond \& Related Materials, 19, 1034-1039. https://doi.org/10.1016/j.diamond.2010.02.014

[10] Strondl, C., van der Kolk, G.J., Hurkmans, T., Fleischer, W., Trinh, T., Carvalho, N.M. and De Hosson, J.Th.M. (2001) Properties and Characterization of Multilayers of Carbides and Diamond-Like Carbon. Surface \& Coatings Technology, 142-144, 707-713. https://doi.org/10.1016/S0257-8972(01)01179-3

[11] Rincon, C., Romero, J., Esteve, J., Martinez, E. and Lousa, A. (2003) Effects of Carbon Incorporation in Tungsten Carbide Films Deposited by r.f. Magnetron Sputtering: Single Layers and Multilayers. Surface \& Coatings Technology, 163-164, 386-391. https://doi.org/10.1016/S0257-8972(02)00635-7

[12] Li, F., Zhang, S., Kong, J., Zhang, Y. and Zhang, W. (2011) Multilayer DLC Coatings via Alternating Bias during Magnetron Sputtering. Thin Solid Films, 519, 4910-4916. https://doi.org/10.1016/j.tsf.2011.01.052

[13] Niu, C., Lu, Y.Z. and Lieber, C.M. (1993) Experimental Realization of the Covalent 
Solid Carbon Nitride. Science, 261, 334-337.

https://doi.org/10.1126/science.261.5119.334

[14] Doemer, M.F. and Nix, W.D. (1986) A Method for Interpreting the Data from Depth-Sensing Indentation Instruments. Journal of Materials Research, 1, 601-609. https://doi.org/10.1557/JMR.1986.0601

[15] Miyake, S. (2003) Improvement of Mechanical Properties of Nanometer Period Multilayer Films at Interfaces of Each Layer. Journal of Vacuum Science and Technology B, 21, 785-789. https://doi.org/10.1116/1.1560217

Submit or recommend next manuscript to SCIRP and we will provide best service for you:

Accepting pre-submission inquiries through Email, Facebook, LinkedIn, Twitter, etc. A wide selection of journals (inclusive of 9 subjects, more than 200 journals)

Providing 24-hour high-quality service

User-friendly online submission system

Fair and swift peer-review system

Efficient typesetting and proofreading procedure

Display of the result of downloads and visits, as well as the number of cited articles Maximum dissemination of your research work

Submit your manuscript at: http://papersubmission.scirp.org/

Or contact msa@scirp.org 